\title{
When less is more: the PI3K pathway as a determinant of tumor response to dietary restriction
}

\author{
Paul J Coffer ${ }^{1}$ \\ ${ }^{1}$ Departments of Immunology \& Pediatric Immunology, University Medical Center Utrecht, Lundlaan 6, 3584 EA Utrecht, The \\ Netherlands \\ Cell Research (2009) 19:797-799, doi: 10.1038/cr.2009.81; published online 2 July 2009
}

For centuries, either due to the scarcity of food or for religious reasons, the beneficial effects of dietary restriction on health and well-being have been appreciated. Reduced calorie intake has been observed to be the most effective way of extending the life-span of mammals without genetically altering them. This increased longevity is also often accompanied by additional health effects, and as long ago as the 1950 s there were reports of caloric restriction $(\mathrm{CR})$ reducing the incidence and growth rate of tumors in laboratory animals [1-3]. However, one of the puzzling aspects of these studies is that responsiveness to a reduction in calorie intake is dependent on the tissue source of the tumor, and not all tumors from the same tissue respond equally well. This suggests that while the growth inhibitory reponse to $\mathrm{CR}$ may be hardwired into all cells, certain tumors have apparently developed escape mechanisms that exempt them from these controls. One of the largely unanswered questions is what is the underlying molecular mechanism that defines how a tumor will respond to reduced calorie intake. A recent study by Kalaany and Sabatini has helped to provide an answer to this [4]. By

Correspondence: Paul J Coffer

E-mail: p.j.coffer@umcutrecht.nl systematically comparing the responsiveness of different types of tumors to $\mathrm{CR}$, they identified phosphatidylinositol 3-kinase (PI3K) as a critical player in the response to $\mathrm{CR}$.

To approach the problem of why only some tumors respond to CR, Kalaany and Sabatini injected human brain and breast cancer cell lines into immunedeficient mice after which a subset of animals were fed $60 \%$ of the calorie intake of their counterparts. While all CR mice exhibited a $20-30 \%$ decrease in body weight, the tumors formed had differential responsiveness ranging from $\mathrm{CR}$-sensitive to CR-resistant. These simple experiments immediately suggest that systemic changes do not underlie the sensitivity of a particular tumor to CR, but rather that other "tumor intrinsic" factors must be involved.

PI3K is an ubiquitously expressed lipid kinase whose activation is critical in the regulation of a multitude of cellular processes. Activation of PI3K by a plethora of hormones, cytokines and growth factors results in production of phosphorylated inositol lipids at the plasma membrane [5]. These lipid second messengers then provide docking sites for a large number of intracellular proteins with a Plekstrin Homology (PH)-domain. The relocation and clustering of $\mathrm{PH}$-domain containing proteins in "signalosomes" subsequently results in the activation of a variety of downstream signaling molecules. Perhaps the best studied of these is Protein Kinase B (PKB/c-akt) whose activity has been implicated in the regulation of proliferation, survival, metabolism and differentiation [6]. Since activation of the PI3KPKB pathway is observed in a wide variety of human cancers, the authors analyzed activation of this signaling module in the various tumor cell lines. Indeed in the CR-resistant cell lines, hyper-activation of PI3K-PKB signaling was observed under in vitro cell culture conditions. This provided the possibility that hyperactivation of PI3K-PKB could possibly override the growth inhibitory effects of $\mathrm{CR}$ (Figure 1).

The PI3K-PKB signaling module is not only hyperactivated in cancer, but also regulates longevity in the nematode worm [7-8]. The Caenorhabditis elegans nematode ages rapidly, undergoing development, senescence, and death in less than 3 weeks. However, worms harboring mutations resulting in inhbition of PI3K-PKB signaling age more slowly than normal and live more than twice as long. Here PKB phosphorylates and inhibits the function of DAF-16, a Forkhead (FOX) transcription factor, whose activation is critical for a specific form of developmental arrest termed "dauer" that is 


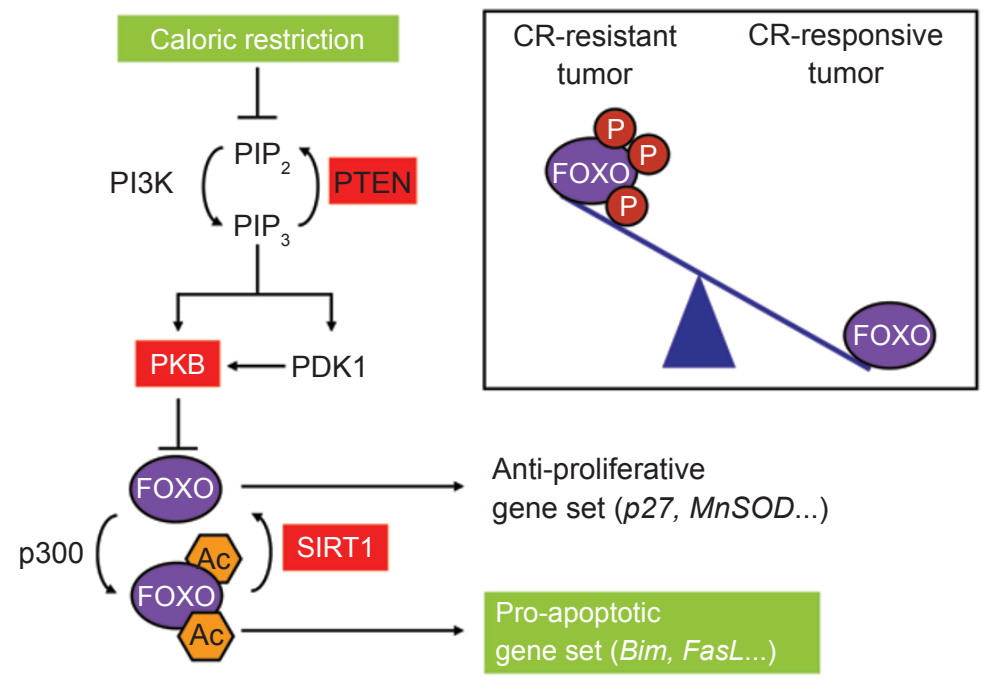

Figure 1 Caloric restriction can reduce tumor size through modulation of FOXO transcription factor activity. Caloric restriction results in reduced PI3K-PKB activity and thus leading to dephosphorylation and nuclear translocation of FOXO transcription factors. Nuclear FOXO activates transcription of pro-apoptotic target genes resulting in increased cell death and a decrease in tumor volume. Deacetylation of FOXO by SIRT1 may prevent the transcription of pro-apoptotic target genes reducing cell death. In CR-resistant tumors, constitutive activation of the PI3K-PKB signaling module prevents activation of FOXO through its phosphorylation and cytoplasmic retention. Pharamcological targeting PI3K, PKB or SIRT1 (marked in red) provides therapeutic options for activating FOXO and increasing apoptosis in CR-resistant tumors.

characterized by lowered metabolic activity and increased lifespan [7-8]. Mammals have three counterparts of DAF-16 which are regulated by PKB and are termed FOXO1, FOXO3 and FOXO4 [9-11]. Phosphorylation of FOXO factors by PKB results in nuclear export thereby repressing transcriptional function. A large number of FOXO target genes have now been described and it is becoming clear that FOXO proteins are critically involved in regulation of proliferation, survival, differentiation, metabolism and cellular response to stress [9-11]. Most recently polymorphisms in $\mathrm{FOXO} 3$ have been shown to correlate with longevity in humans suggesting an evolutionary conserved role for this transcription factor [12].

Hyperactivation of PI3K-PKB signaling in $\mathrm{CR}$ resistant tumors would be expected to be coupled with decreased FOXO transcription factor activity. This was borne out by increased phosphorylation and cytoplasmic localization of FOXO1 in CR-resistant tumor cells. In contrast, in CR-sensitive tumors, dietary restriction resulted in a relocalization of FOXO1 from nucleus to cytoplasm demonstrating reduced PI3K-PKB activity. Furthermore, these tumor cells exhibited increased levels of apoptosis after starvation. Taken together, these data point towards a model where increased PI3K-PKB activity and decreased FOXO-mediated transcription are a hallmark of tumors that are insensitive to CR. This was further supported by performing xenograft studies in which isogenic co-rectal cell lines either expressing wild-type or constitutively active PI3K were analyzed for growth responses after CR. In these experiments the tumors expressing active PI3K were resistant to dietary restriction in contrast to their isogenic counterparts. Thus the introduction of a PI3K-activating mutation in an otherwise CR-sensitive tumor is sufficient to induce resistance to dietary restriction (Figure 1).

So why would the activation status of PI3K determine the response of a tumor to dietary restriction? To investigate this, Kalaany and Sabatini further examined the role of FOXO1 in tumor sensitivity. As previously mentioned, this transcription factor family has already been implicated in regulating response to $\mathrm{CR}$ in both lower and higher eukaryotes [9]. Over-expression of FOXO1 resulted in the reduction of tumor volume after starvation in a CR-resistant colon carcinoma cell line. This was accompanied not by decreased proliferation but by increased apoptosis of tumor cells. FOXO transcription factors have been demonstrated to be actively involved in inducing apoptosis through both mitochondrial-dependent and -independent mechanisms [13]. This occurs at multiple levels including increasing expression of death receptor ligands FasL and TRAIL, as well as the proapoptotic Bcl-2 family member Bim [13]. Although expression levels of these proteins were not investigated in CR-sensitive tumor cell lines, it is likely that such mechanisms are responsible for increased apoptosis after dietary restriction.

As gate-keepers regulating cellular survival and proliferation, FOXO factors are subject to multiple levels of regulation. SIRT1 is an NAD-dependent deacetylase that deacetylates FOXO transcription factors leading to modulation of their transcriptional function [14]. SIRT1 is thought to play a broad biological role in the regulation of growth, stress response, tumorigenesis and lifespan [15]. Although the precise role of SIRT1 in regulating FOXO-mediated transcription remains incompletely defined, it 
has been proposed that in mammals SIRT1 may shift responses away from apoptosis, and towards cellcycle arrest and survival. When overexpressed, Sir 2 , the closest $C$. elegans homologue of SIRT1, extends the lifespan of the worm in a daf-16dependent manner [15]. This suggests that targeting FOXO deacetylation through SIRT-inhibition could provide an additional therapeutic strategy to promote apoptosis in CR responsive tumors.

While the study by Kalaany and Sabatini provides insight into the molecular mechanisms that determine whether a tumor is responsive or not to dietary restriction, the clinical implications of these findings remain unclear. However, for the first time this study provides a relatively simple means of predicting whether a tumor would be responsive to CR or not. Pharmacological inhibition of the PI3K-PKB signaling module is the subject of intensive investigation by many companies and academic centers, and many PI3K-pathway targeted inhibitors are currently being tested in pre-clinical and clinical trials [5]. It is possible that limiting calorie intake in combination with such inhibitors could provide extra benefit to patients with CR-responsive tumors.

\section{References}

1 McCay CM, Crowell MF, Maynard LA. The effect of retarded growth upon the length of lifespan and upon the ultimate body size. J Nutr 1935; 10:63-79.

2 Tannenbaum A, Silverstone H. The influence of the degree of caloric restriction on the formation of skin tumors and hepatomas in mice. Cancer Res 1949; 9:724-727.

3 Weindruch R, Walford R. Dietary restriction in mice beginning at 1 year of age: effect on life-span and spontaneous cancer incidence. Science 1982; 215:1415-1418.

4 Kalaany NY, Sabatini DM. Tumours with PI3K activation are resistant to dietary restriction. Nature 2009; 458:725731.

5 Hennessy BT, Smith DL, Ram PT, Lu Y, Mills GB. Exploiting the PI3K/AKT pathway for cancer drug discovery. Nat Rev Drug Disc 2005; 4:988-1004.

6 Franke TF. PI3K/Akt: getting it right matters. Oncogene 2008; 27:64736488.

7 Lin K, Dorman JB, Rodan A, Kenyon C. daf-16: An HNF-3/forkhead family member that can function to double the life-span of Caenorhabditis elegans.
Science 1997; 278:1319-1322.

8 Ogg S, Paradis S, Gottlieb S, et al. The Fork head transcription factor DAF-16 transduces insulin-like metabolic and longevity signals in C. elegans. Nature 1997; 389:994-999.

9 Salih DA, Brunet A. FoxO transcription factors in the maintenance of cellular homeostasis during aging. Curr Opin Cell Biol 2008; 20:126-136.

10 Maiese K, Chong ZZ, Shang YC. OutFOXOing disease and disability: the therapeutic potential of targeting FoxO proteins. Trends Mol Med 2008; 14:219-227.

11 van der Horst A, Burgering BM. Stressing the role of FoxO proteins in lifespan and disease. Nat Rev Mol Cell Biol 2007; 8:440-450.

12 Flachsbart F, Caliebe A, Kleindorp R, et al. Association of FOXO3A variation with human longevity confirmed in German centenarians. Proc Natl Acad Sci USA 2009; 106:2700-2705.

$13 \mathrm{Fu} \mathrm{Z}$, Tindall DJ. FOXOs, cancer and regulation of apoptosis. Oncogene 2008; 27:2312-2319.

14 Giannakou ME, Partridge L. The interaction between FOXO and SIRT1: tipping the balance towards survival. Trends Cell Biol 2004; 14:408-412.

15 Kim EJ, Um SJ. SIRT1: roles in aging and cancer. BMB Rep 2008; 41:751756. 